\title{
LGR5, a novel functional glioma stem cell marker, promotes EMT by activating the $W n t / \beta$-catenin pathway and predicts poor survival of glioma patients
}

\author{
Jin Zhang ${ }^{1}$, Hongqing Cai ${ }^{1}$, Lixin Sun ${ }^{2}$, Panpan Zhan ${ }^{2}$, Meng Chen², Feng Zhang ${ }^{2}$, Yuliang Ran ${ }^{2 *}$
} and Jinghai Wan ${ }^{1 *}$

\begin{abstract}
Background: Tumor recurrence, the chief reason for poor prognosis of glioma, is largely attributed to glioma stem cells (GSCS) and epithelial-mesenchymal transition (EMT). However, the mechanisms among them remain unknown. Here, we determined whether leucine-rich repeat-containing G protein-coupled receptor 5 (LGR5), known as a stem cell marker for colon cancer and gastric cancer, can serve as a novel GSC marker involved in EMT and a therapeutic target in glioma.

Methods: Stemness properties were examined in FACS-isolated LGR5 ${ }^{+} / \mathrm{LGR5} 5^{-}$cells. Reported stem cell markers, EMT and the Wnt/B-catenin pathway were examined in stable LGR5 knockdown or overexpressed GSCs by Western Blot. The treatment experiment was performed in an intracranial orthotopic xenograft model by knockdown of LGR5 or by using the Wnt/ $\beta$-catenin pathway inhibitor Wnt-C59. LGR5 expression was determined in 268 glioma specimens by immunohistochemistry.

Results: $\mathrm{LGR5} 5^{+}$cells possessed stronger stemness properties compared to $\mathrm{LGR}^{-}$cells. The expression of SOX2, Nanog, CD133, CD44, CD24 and EpCAM was modulated by LGR5. Both LGR5 knockdown and Wnt-C59 reduced tumor invasion and migration and blocked EMT by inhibiting the Wnt/B-catenin pathway in vitro and suppressed the intracranial orthotopic xenograft growth and prolonged the survival of xenograft mice in vivo. Moreover, LGR5 was positively correlated with Ki67, N-cadherin and WHO grade and negatively correlated with IDH1. Glioma patients with high expression of LGR5 showed significantly poorer prognosis.

Conclusions: LGR5 is a new functional GSC marker and prognostic indicator that can promote EMT by activating the Wnt/ $\beta$-catenin pathway and would thus be a novel therapeutic target for glioma.
\end{abstract}

Keywords: Glioma stem cell, LGR5, EMT, Wnt/ß-catenin, Glioma recurrence, Glioma survival

\section{Background}

Glioma is the most common and aggressive primary brain tumor [1]. Depending on the malignancy grade, gliomas can be classified into four levels from I to IV according to World Health Organization (WHO) criteria [2]. Despite multiple

\footnotetext{
*Correspondence: ran_yuliang@126.com; wanjinghai@sina.com

${ }^{2}$ Chinese Academy of Medical Sciences and Peking Union Medical College, National Cancer Center/National Clinical Research Center for Cancer/Cancer Hospital, Beijing 100021, China

${ }^{1}$ Department of Neurosurgery, Chinese Academy of Medical Sciences and Peking Union Medical College, National Cancer Center/National Clinical Research Center for Cancer/Cancer Hospital, Beijing 100021, China
}

rigorous treatments comprising maximal surgical resection, radiotherapy, and chemotherapy, the prognosis remains dismal, with a median overall survival time of approximately 15 months for glioblastoma multiforme (GBM, WHO grade IV) $[1,3]$. Epithelial-mesenchymal transition (EMT) is a vital process through which epithelial cells lose cell polarity and adhesiveness and thus transform into mesenchymal cells, which have an increased invasive or metastatic phenotype [4]. This high capacity for invasion and migration caused by EMT often limits total surgical resection and contributes to therapeutic resistance, which eventually leads to tumor 
recurrence [3-6]. However, the mechanism of signaling pathways and effector molecules that drive glioma EMT and invasion remains not well knowable [4]. Recent developments in stem cell research have revealed the existence of glioma stem cells (GSCs) which are analogous to cancer stem cells (CSCs) and determined that GSCs represent a subpopulation of cells with strong tumorigenesis, invasiveness, chemoradiotherapy resistance and EMT properties [7, 8]. Precisely because of these characteristics, GSCs are considered as responsible for failure of treatment and tumor recurrence [8-12]. Therefore, GSCs are regarded as a relevant target for glioma therapy.

Accumulated evidences have suggested that induction of EMT induces CSC phenotype in tumor cells $[13,14]$ or human mammary epithelial cells and gives rise to invasive and metastatic CSCs $[15,16]$. These findings indicated that the mechanisms for regulating EMT and stemness may be closely integrated [17-19]. However, the underlying mechanisms between GSCs and EMT are not fully understood. Therefore, searching for the association between GSCs and EMT may have significant implications for the exploration of both the causes of glioma recurrence and subsequent therapeutic targets.

The therapeutic strategy targeting CSCs is mainly focused on the direct elimination of CSCs by targeting cell surface markers or specific pathways that are essential for maintaining stemness properties $[10,20]$. Although some of CSC markers cluster of differentiation (CD) 133, CD44, CD24, CD90 and epithelial cell adhesion molecule (EpCAM), have been employed to identify in GSCs [10, 21, 22], their functionalities remain defective $[20,23,24]$. Moreover, few such surface markers have been confirmed to be functional in GSCs. Leucine-rich repeat-containing $G$ protein-coupled receptor 5 (LGR5), a seven-transmembrane receptor of the G protein-coupled receptor family related to the Wnt pathway, was initially identified as a marker of intestinal stem cells [25]. LGR5 has been reported as a stem cell marker in several tumors [26-29]. Studies have shown that LGR5 can promote tumor initiation, proliferation and invasion [26, 29-32]. Nakata et al. demonstrated that LGR5 level is positively correlated with pathologic grade and an adverse outcome in glioma [33]. However, the role of LGR5 in GSCs has not yet been determined. Moreover, the relevance of LGR5 to invasion and EMT in glioma remains unknown. Hence, we aimed to investigate whether LGR5 is associated with GSC properties and its relationship with EMT and the Wnt pathway in GSCs.

In this study, we determined the stemness properties of $\mathrm{LGR}^{+}$and $\mathrm{LGR}^{-}$glioma cells sorted by fluorescence-activated cell sorting (FACS). The relationship between LGR5 and known CSC markers were examined by flow cytometry (FCM) and Western blot. Using a lentiviral vector and $\mathrm{Wnt} / \mathrm{\beta}$-catenin pathway inhibitor Wnt-C59, we explored the association of LGR5 with EMT and Wnt/ $\beta$-catenin pathway in GSCs in vitro and in vivo. Moreover, the LGR5 expression was determined in human glioma tissues. The role of LGR5 in GSCs was systematically investigated in biological and clinical conditions, which may provide novel insights into the biological progress of GSCs and aid in the development of evaluation of clinical prognosis and targeted GSCs therapy.

\section{Methods \\ Patients}

Patients with a history of other malignant tumor and preoperative adjuvant chemotherapy and/or radiotherapy as well a cause of death unrelated to glioma were excluded from the current study. A total of 268 adult glioma patients who had undergone total tumor resection at the Cancer Hospital Chinese Academy of Medical Sciences between January 2012 and December 2016 were included in this retrospective study. The histological pathologies of all patients were confirmed by at least three experienced pathologists and categorized as low-grade (WHO I-II, $n=85$ ) or high-grade (WHO III-IV, $n=183$ ) according to WHO criteria. The degree of peritumoral edema was retrospectively assessed by T2W imaging from 268 glioma patients and was divided into two groups according to the maximum diameter of the (maximum diameter $>2 \mathrm{~cm}$, severe; maximum diameter $\leqq 2 \mathrm{~cm}$, slight/None). Overall survival (OS) and progression-free survival (PFS) were investigated and recorded from the date of the first surgery to the date of death or the endpoint of this study (September 2, 2017). The clinicopathological characteristics of glioma patients are outlined in Additional file 1: Table S1 and Additional file 2: Table S2. This study was approved by the Ethics Committee of Cancer Hospital, Chinese Academy of Medical Sciences. Written informed consent for the use of resected specimens was prospectively obtained from all patients.

\section{Primary glioma cells}

Fresh glioma specimens (shown as glioma by intraoperative frozen pathology and confirmed by postoperative paraffin pathology) were immediately placed in ice-cold DMEM to slow cell metabolism, avoiding excessive damage at room temperature in vitro. Then, the tissues were washed with DMEM, removed from the blood vessels, minced and dissociated into approximately $1 \mathrm{~mm}$ pieces by ophthalmic scissors. The samples were then triturated by a homogenizer (Gentle MACS Dissociator) for 10 min; resuspended in DMEM containing 20\% FBS, $1 \%$ L-glutamine, and 1\% penicillin/streptomycin; and passed through a cell strainer. The filtrate containing cells was maintained at a density of $2 \times 10^{5}$ cells $/ \mathrm{ml}$ in a cell culture incubator with $5 \% \mathrm{CO}_{2}$ at $37{ }^{\circ} \mathrm{C}$. The entire process was completed in $45 \mathrm{~min}$ without trypsin. 


\section{GSCs enriched from glioma cells}

Human glioma cell lines (U251, U87MG, A172) were obtained from the Basic Medical Research Institute of the Chinese Academy of Medical Science (October, 2015). Human primary glioma cells (8591, 7112 and LHH) were derived from glioma specimens of the Cancer Hospital, Chinese Academy of Medical Sciences. The main glioma cells used for this study (U251 and 8591) were authenticated by short tandem repeat (STR) profiling (see Additional files 3 and 4).

Before the enrichment experiment, all parent glioma cells were cultured in DMEM (Invitrogen) with 10\% FBS (Invitrogen). To obtain enriched cells, the medium of the parent cells was replaced with a serum-free medium containing neurobasal (Invitrogen) supplemented with $40 \mathrm{ng} / \mathrm{ml}$ human fibroblast growth factor (hFGF, Peprotech), $40 \mathrm{ng} / \mathrm{ml}$ human epidermal growth factor (hEGF, Peprotech) and 2\% B27 (Invitrogen) during cell generation. After 7 days, the parent cells and the enriched cells were respectively digested and dissociated as single cells to perform the FCM.

\section{Flow cytometry for detection and sorting}

Parent cells and enriched cells were incubated with LGR5 (\#ab75735; Abcam) and an isotype control (\#ab171870; Abcam) and subsequently with secondary antibodies (Alexa Fluor 488) for $30 \mathrm{~min}$ at room temperature and washed three times in phosphate buffered saline (PBS) after each reaction to detect the proportion of LGR5 expression. Using the method described above, LGR $5^{+}$and LGR5 ${ }^{-}$glioma cells were sorted from parent cells by FACS.

To explore the proportion of reported stem cell markers in both $\mathrm{LGR}^{+}$and LGR5 ${ }^{-}$glioma cells, the cells were incubated with APC anti-human CD133 antibody (\#130090-826; Miltenyi), APC anti-human CD44 antibody (\#170441-82; eBioscience), APC anti-human CD24 antibody (\#311118; BioLegend), APC anti-human CD90 antibody (\#328114; BioLegend) or APC anti-human EpCAM (\#328208; BioLegend) antibody for $30 \mathrm{~min}$ at room temperature. APC anti-human IgG (\#409306; BioLegend) was used as an isotype control for these markers. All stained cell suspensions were detected or sorted using an AccuriC6 cytometer (BD Biosciences) and analyzed by Flowjo 7.6.1.

\section{Proliferation, clone formation and drug resistance assay}

The sorted LGR $5^{+}$and $\mathrm{LGR} 5^{-}$cells were seeded into 96-well culture plates at a density of $1 \times 10^{3}$ cells per well in $100 \mu \mathrm{L}$ of medium with $10 \%$ FBS. The cells were then cultured for 1-5 days, and the cell numbers were determined by a Cell Counting Kit-8 (CCK8, Dojindo) assay according to the manufacturer's instructions. Then, $10 \mu \mathrm{L}$ of CCK8 solution was added to each well of the plate for $1 \mathrm{~h}$ of incubation and the absorbance was measured using a microplate reader at $450 \mathrm{~nm}$.
To examine the ability of clone formation, $600 \mu \mathrm{L}$ of serum-free medium supplemented with $0.8 \%$ methyl cellulose, which contained $500 \mathrm{LGR}^{+}$or LGR5 ${ }^{-}$cells, was added to each well of the 24-well ultra-low attachment plates (Corning). Every 5 days, we replenished the medium and the colonies were counted after 14 days. The tumor spheres larger than $50 \mu \mathrm{m}$ in diameter were evaluated in this study.

Temozolomide (TMZ, Sigma) was dissolved in dimethyl sulfoxide (DMSO; Sigma) and a final DMSO concentration was diluted to under $0.5 \%$ by $10 \%$ FBS DMEM medium. LGR $5^{+}$and LGR $5^{-}$cells were cultured with TMZ for $72 \mathrm{~h}$ at concentrations of $0,25,50,100$, 200, 400 and $800 \mu \mathrm{M}$. The absorbance was detected using CCK8 assays by a microplate reader at $550 \mathrm{~nm}$. The $\mathrm{IC}_{50}$ was calculated by Graphpad 5.0. Each aforementioned assay was performed in triplicate.

\section{Lentiviral transfection}

All lentiviral vectors for LGR5 knockdown and overexpression were designed and synthesized by Genecopoeia Ltd. (Guangzhou, China), and expressed green fluorescent protein (GFP) and puromycin-resistant proteins for screening. The target sequence of the LGR5 shRNA (shLGR5) was 5'-GCTCTCATCTTGCTCAATTCC-3', and the target sequence of negative control shRNA (shCtrl) was 5'-GCTTCGCGCCGTAGTCTTA-3'. LGR5 is overexpressed in glioma cells with the transfection of lentivirus containing LGR5 (Lenti-LGR5). Glioma cells infected with lentivirus containing empty vectors were used as negative controls (Lenti-GFP). The transfection process was performed according to the manufacturer's protocol. The multiplicity of infection (MOI) was 5 for U251 and 10 for 8591 . Stably transfected cells were selected by addition of $1 \mu \mathrm{g} / \mathrm{ml}$ of puromycin (Gibco) at 3 days after infection for 1 week. The transfection efficiency was confirmed by FACS and Western blot.

\section{Invasion and migration assays}

The invasion of glioma cells was assessed in transwell chambers $(8-\mu \mathrm{m}$ pore size, Miltenyi) coated with Matrigel (BD Biosciences; 1:4 volume) in accordance with the manufacturer's protocol. A total of $3 \times 10^{4}$ glioma cells suspended in $100 \mu \mathrm{L}$ of serum-free DMEM were added onto the upper chamber of a transwell plate, and $600 \mu \mathrm{L}$ of normal culture medium of $10 \%$ FBS was added to the lower chamber. After $24 \mathrm{~h}$ of incubation, the cells on the upper surface of the chamber were removed, and the cells on the lower surface were fixed by methanol for $15 \mathrm{~min}$, stained by $1 \%$ crystal violet for $30 \mathrm{~min}$ and counted. To investigate the effect of Wnt-C59 (APExBio) on the invasion and migration GSCs, Wnt-C59 was completely dissolved in DMSO with a concentration of $2.0 \times 10^{4} \mu \mathrm{M}$, and the GSCs were divided into three 
groups (a control group disposed with DMSO, and two experimental groups disposed with $5 \mu \mathrm{M}$ Wnt-C59 or $20 \mu \mathrm{M}$ Wnt-C59), which were all incubated for $24 \mathrm{~h}$. The media of the transwell experiment in both the upper and lower chambers were all disposed with the same concentration as described above. Moreover, the concentrations of DMSO in the media of three groups were guaranteed to be equal. To assess the migration of GSCs, all the procedures were consistent with invasion except that the chamber was not covered with Matrigel and the incubation time was shortened to $8 \mathrm{~h}$. Each assay was performed in triplicate.

\section{Intracranial and subcutaneous xenograft models and magnetic resonance imaging (MRI)}

All animal procedures were approved by the Experimental Animal Ethics Committee of the Cancer Hospital, Chinese Academy of Medical Sciences. For the establishment of intracranial xenograft models, male 4- to 5-week-old BALB/c-nu mice (Beijing HFK Bioscience Ltd) were stabilized with a stereotactic apparatus (KOPF940) with continuous anesthetization with isoflurane (RWD Life Science). Then the shCtrl and shLGR5 GSCs were injected into the caudate nucleus of brain as previously described with $5 \times 10^{5}$ cells in $3 \mu \mathrm{L}$ of DMEM/mice for U251 or $1 \times$ $10^{6}$ cells in $5 \mu \mathrm{L}$ DMEM/mice for 8591 . After injection, 5 of $10 \mathrm{shCtrl}$ mice were treated with $200 \mu \mathrm{L}$ Wnt-C59 (15 mg/kg/day) by oral administration. Then, the intracranial tumor of T2-weight (T2W) images of the shCtrl ( $n=$ $5)$, shLGR5 $(n=5)$ and Wnt-C59 $(n=5)$ groups were examined by a 7.0 T MRI scanner (Bruker BioSpin, Billerica, MA, USA) with a relaxation enhancement (RARE) pulse sequence $(\mathrm{TR}=4030 \mathrm{~ms}, \mathrm{TE}=50 \mathrm{~ms}$, matrix $=320 \times 384$, slice thickness $=0.3 \mathrm{~mm}$ ). The maximal anteroposterior diameter $(\mathrm{L})$, transverse diameter $(\mathrm{W})$ and height $(\mathrm{H})$ were measured with a RadiAnt DICOM Viewer. Tumor volume was calculated with the following formula: $\mathrm{V}=\pi / 6 \times \mathrm{L} \times$ $\mathrm{W} \times \mathrm{H}\left(\mathrm{mm}^{3}\right)$.

For the establishment of subcutaneous xenograft models, the mice and the anesthesia method were the same as with the intracranial xenograft models. The sorting glioma cells suspended in $100 \mu \mathrm{L}$ of DMEM were injected directly into the dorsal areas (left for $\mathrm{LGR}^{-}$and right for $\mathrm{LGR}^{+}$) of mice with $5 \times 10^{5}$ cells/ mice for U251 or $1 \times 10^{6}$ cells/mice for 8591 . We measured the tumor size every 3 days and photographed at the endpoint of the experiment.

\section{Western blot}

Proteins were extracted using a Total Protein Extraction Kit (Invent Biotechnologies, SD-001) according to the manufacturer's instruction. Western blot analyses were performed as previously reported [34]. The following primary antibodies were used to detect protein expression:
LGR5 (\#LS-C98619; Biosciences), CD133 (\#ab19898; Abcam), CD24 (\#ab179821; Abcam), EpCAM (\#ab71916; Abcam), CD44 (\#3570; Cell Signaling), N-cadherin (\#13116; Cell Signaling), E-cadherin (\#14472; Cell Signaling), SOX2 (\#3579; Cell Signaling), Nanog (\#3580; Cell Signaling), OCT4 (\#2750; Cell Signaling), Vimentin (\#3932; Cell Signaling), $\beta$-catenin (\#8480; Cell Signaling),

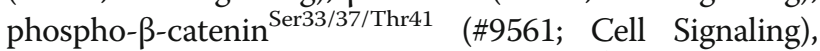
Non-phospho (Active) $\beta$-catenin Ser33/37/Thr41 (Active- $\beta$-catenin) (\#8814; Cell Signaling) and $\beta$-actin (\#4970; Cell Signaling). The dilution was performed according to the antibody specification.

Immunohistochemistry (IHC) and immunofluorescence (IF) IHC staining of human glioma specimens and subcutaneous or intracranial tumors were performed as previously described [34]. All antibodies used were as follows: LGR5 (\#TA502948; OriGene), Ki67 (\#ab16667; Abcam), CD44 (\#15675-1-AP, Proteintech), SOX2 (\#ab92494; Abcam), Non-phospho (Active) $\beta$-catenin Ser33/37/Thr41 (\#8814; Cell Signaling) and N-cadherin (\#bs-1172R; Bioss). For quantitative analysis of IHC, each slice was randomly photographed from five different fields. The expression levels of LGR5, Ki67, Active- $\beta$-catenin, and $\mathrm{N}$-cadherin were analyzed using Image-Pro Plus 5.0. For evaluation of IHC staining of LGR5, the IHC intensity and the expression level of LGR5 were independently reviewed by two observers in a blinded manner. The IHC score was calculated by multiplying the IHC staining intensity and the percentage of positive tumor cells. The staining intensity was defined as follows: $0=$ no staining; $1=$ weak staining; $2=$ medium staining; 3 =strong staining. The percentage of positive tumor cells as graded as 1 for $0-25 \%, 2$ for $26-50 \%, 3$ for $51-75 \%$ and 4 for $>75 \%$. The final score of LGR5 staining was used to determine the expression level of LGR5, which was categorized into low expression group $\left(\right.$ LGR $5^{\text {low }}$, total score $\left.\leqq 4\right)$ and high expression group $\left(\right.$ LGR $5^{\text {high }}$, total score $>5$ ).

IF staining were performed as previously described [35]. The primary antibodies used were as follows: LGR5 (\#LS-C98619; Biosciences), LGR5 (\#TA502948; OriGene), GFAP (\#ab190288; Abcam), SOX2 (\#3579; Cell Signaling) and N-cadherin (\#13116; Cell Signaling). Alexa Fluor 488 (\#4412; Cell Signaling) and Alexa Fluor 647 (\#4410; Cell Signaling) were used as the secondary antibody at a dilution of 1:500. IF images were captured with a confocal microscope (BD Biosciences).

\section{Statistical analysis}

Statistical analysis was performed using GraphPad Prism 5.0 (GraphPad Software Inc.) and SPSS version 19.0 (SPSS Inc.). Two-tailed Student $t$ test was used to analyze the differences in the results between groups. 
Comparisons among three or more groups were assessed using a one-way analysis of variance (ANOVA). Comparison between two or more groups in different time points were assessed by two-way ANOVA. Correlations between LGR5 and Ki67, N-cadherin, SOX2 and CD44 expressions were analyzed by Spearman correlation method. All values are expressed as means \pm SD. The correlation between LGR5 expression and clinicopathological variables was analyzed by a $\chi^{2}$ test or Fisher's exact test. OS and PFS curves were plotted by the Kaplan-Meier method and compared using the results of a Log-rank test. The Cox proportional hazards model was used to estimate the independent prognostic factors for OS and PFS in the multivariate analysis. $P$ values less than 0.05 were considered statistically significant.

\section{Results}

Percentage of LGR5 ${ }^{+}$cells is higher in enriched cells than that in parent cells

To determine the expression and localization of LGR5 in glioma cells, LGR5 staining was performed in 3 types of glioma cell lines (U251, U87 and A172) and 3 types of human primary glioma cells (8591, LHH and 7112), demonstrating that LGR5 was expressed in the cell membrane and cytoplasm (Fig. 1a). All of the abovementioned glioma cells were proved to be derived from astrocytes by glial fibrillary acidic protein (GFAP) co-dyeing (Fig. 1a). We obtained enriched cells from parent cells through serum-free enrichment, which is a method for quickly screening GSCs. The expression of LGR5 was detected in glioma parent cells and in enriched cells by FCM (Additional file 5: Figure S1a). The positive proportions of LGR5 were $2.46 \%, 2.01 \%$, $5.76 \%, 1.34 \%, 1.79 \%$ and $1.45 \%$ in U251, U87, A172, 8591, LHH and 7112 parent cells, respectively, and $21.50 \%, 11.23 \%, 16.04 \%, 15.42 \%, 11.41 \%$ and $4.53 \%$ in U251, U87, A172, 8591, LHH and 7112 enriched cells, respectively. The positive rates of LGR5 in enriched cells were 8.7, 5.6, 2.8, 11.5, 6.4 and 3.1 times higher than those in parent cells for U251, U87, A172, 8591, LHH and 7112, respectively. Thus, we chose U251 and 8591, whose enrichment levels were the highest (Fig. 1b), to establish cell models. Then, $\mathrm{LGR}^{+}$and $\mathrm{LGR}^{-}$cells were obtained by FACS to perform follow-up experiments (Additional file 5: Figure S1b).

\section{LGR5 ${ }^{+}$cells possess higher proliferation and clone formation abilities}

Compared with LGR5 ${ }^{-}$cells, LGR5 ${ }^{+}$U251 cells possessed higher proliferation activity $(P<0.001, n=3$, Fig. $1 c)$ and presented a higher ability to form colonies $(P<0.01, n=3$, Fig. 1d). Moreover, the clone formation assay showed that $\mathrm{LGR}^{+}$U251 cells formed larger spheres, whereas the LGR5 ${ }^{-}$U251 cells formed smaller spheres at day 14 (Fig. 1d). Similar results were obtained for 8591 (Additional file 6: Figure $\mathrm{S} 2 \mathrm{a}, \mathrm{b})$. These results indicated that LGR $5^{+}$cells exhibited stronger tumor initiation and cloning abilities compared to LGR5 $5^{-}$cells in vitro.

\section{$\mathrm{LGR5}^{+}$cells are more resistant to temozolomide (TMZ)}

GSCs usually show strong resistance to chemotherapy. TMZ is the foremost medicine for clinical adjuvant treatment of glioma; therefore, we tested the resistance of $\mathrm{LGR}^{+}$and $\mathrm{LGR}^{-}$cells to TMZ. The $\mathrm{IC}_{50}$ values were $142.5 \mu \mathrm{M}$ for LGR5 ${ }^{-} \mathrm{U} 251$ cells and $376.0 \mu \mathrm{M}$ for LGR5 ${ }^{+}$ U251 cells ( $n=3$, Fig. 1 e) and were $256.8 \mu \mathrm{M}$ for LGR5 ${ }^{-}$ 8591 cells and $471.3 \mu \mathrm{M}$ for LGR ${ }^{+} 8591$ cells (Additional file 6: Figure S2c). LGR5 ${ }^{+}$glioma cells expressed stronger resistance to TMZ than $\mathrm{LGR}^{-}$glioma cells.

LGR5 $^{+}$cells show higher ability of invasion and migration We performed invasion and migration assays of LGR $5^{+}$and LGR5 ${ }^{-}$U251 cells and found that LGR5 ${ }^{+}$U251 cells were significantly more invasive and migratory than LGR5 ${ }^{-}$cells $(P<0.001, n=3 ; P<0.001, n=3$, respectively, Fig. 1f $)$. Similar results were observed in 8591 primary glioma cells (Additional file 6: Figure S2d). Then, we examined the expression of LGR5 and EMT activated marker N-cadherin in LGR5 $^{+}$and LGR5 ${ }^{-}$cells by Western blot. The results clearly showed higher expression levels of LGR5 and $\mathrm{N}$-cadherin in LGR5 ${ }^{+}$cells (Fig. 1g), suggesting that LGR5 may be related to invasiveness and EMT in glioma.

\section{Xenograft tumors derived from $\mathrm{LGR}^{+}$cells are more malignant and invasive, exhibiting strong stemness phenotype}

Tumorigenic ability in vivo is known to be the gold standard for identifying GSCs. We transplanted $5 \times 10^{5}$ LGR $^{+}$ and $5 \times 10^{5}$ LGR $^{-}$U251 cells into the backs of BALB/c-nu mice. The xenografts produced by the LGR $5^{+} \mathrm{U} 251$ cells (LGR5 $^{+}$xenografts) were larger than those xenografts produced by the LGR5 ${ }^{-}$U251 cells (LGR5 ${ }^{-}$xenografts) $(P<$ $0.001, n=5$, Fig. 2a, b). In addition, IHC was performed to validate the role of LGR5 in the malignancy and invasiveness and stemness of glioma xenografts. The expression of LGR5 was remarkably higher in LGR $5^{+}$xenografts than that in LGR5 $5^{-}$xenografts $(P<0.01, n=5$, Fig. 2 c, d). The expression level of Ki67, a sign of proliferation for tumor malignancy, was higher in LGR $5^{+}$xenografts than that in LGR5 $^{-}$xenografts $(P<0.05, n=5$, Fig. 2c, d). Moreover, the LGR $5^{+}$xenografts exhibited significantly higher expression of N-cadherin compared with the LGR5 ${ }^{-}$xenografts $(P<$ $0.01, n=5$, Fig. 2c, d), consistent with the previous Western blot analysis in LGR5 ${ }^{+}$U251 cells. Notably, the CSCs markers levels of both SOX2 and CD44 were also found to be overexpressed in $\mathrm{LGR}^{+}$xenografts $(P<0.01, n=5 ; P<$ $0.01, n=5$, respectively, Fig. 2c, d). To verify whether LGR5 level correlated with these tumor markers in glioma, 


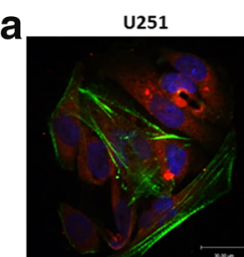

8591



LHH


d



f

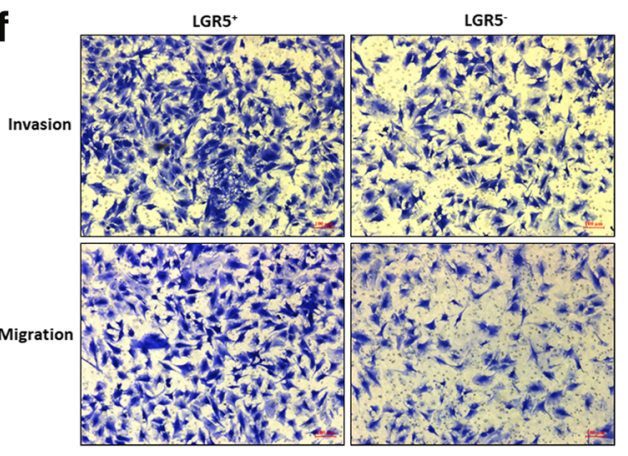

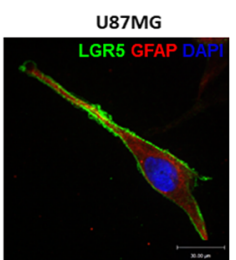

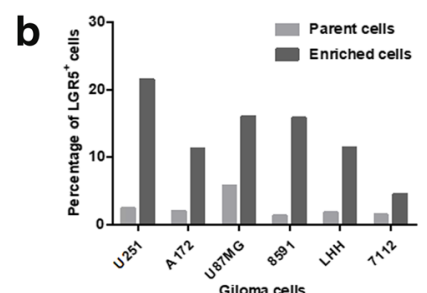

C



e

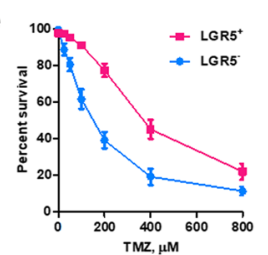

g
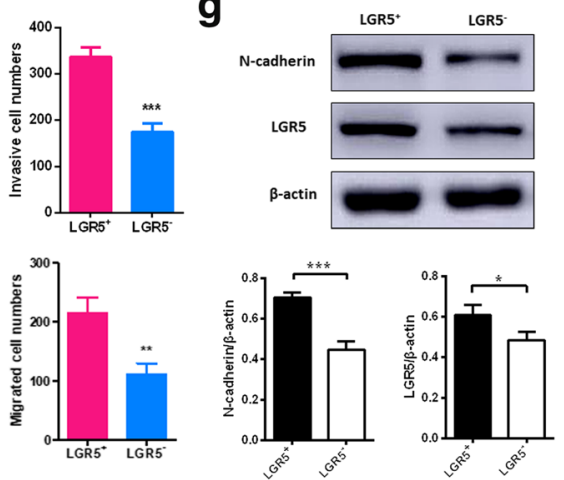

Fig. 1 LGR5 expression in different glioma cells, and stemness properties of LGR5 ${ }^{+}$U251 cells in vitro. a The expression and localization of LGR5 and GFAP in the glioma cell lines (U251, A172 and U87MG) and the human primary glioma cells (8591, LHH and 7112). Scale bar = 30 $\mu$ m. b The enrichment levels of LGR5 expression by FCM in parent cells and enrichment cells. c Cell proliferation assays of LGR5 ${ }^{+}$U251 cells and LGR5 ${ }^{-}$U251 cells $(P<0.001, n=3$, two-way ANOVA). d Coloning sphere images and sphere numbers in the clone formation assay $(P<0.01, n=3$, Student $t$ test). Scale bar $=100 \mu \mathrm{m}$. e Drug resistance curve of TMZ in LGR5 ${ }^{+}$and LGR5 $\mathrm{U} 251$ cells. $\mathbf{f}$ Images and numbers of invasive cells in invasion assays (top, $P<0.001, n=3$, Student $t$ test) and images and numbers of migrated cells in migration assays (bottom, $P<0.001, n=3$, Student $t$ test). $\mathbf{g}$ Western blot analysis for LGR5 and N-cadherin in LGR5 $5^{+}$and LGR5 $5^{-}$U251 cells. The expression levels of LGR5 and N-cadherin were quantified by Image lab software by densitometric analysis and were normalized to the control groups. Human $\beta$-actin was used as the internal control. ( $P<0.05, n=3 ; P<0.001, n=3$, respectively, Student $t$ test). All data are represented as mean $\pm \mathrm{SD}$ from triplicate wells. ${ }^{*}, P<0.01$; ***, $P<0.001$, as compared to control

Spearman correlation analyses were performed. Significant positive correlations were observed between the LGR5 level and both Ki67 $(P<0.01, r=0.7794, n=10$, Fig. 2e) and $\mathrm{N}$-cadherin level $(P<0.01, r=0.8424, n=10$, Fig. $2 \mathrm{f})$, as well as SOX2 level $(P<0.001, r=0.8788, n=10$, Fig. $2 \mathrm{~g})$ and CD44 level ( $P<0.05, r=0.6970, n=10$, Fig. 2h). Additionally, the co-expression of LGR5 and N-cadherin were observed mostly in the peripheral xenograft edge, while positive expression is rare in inside xenografts (Fig. 2i). Similar results were obtained for 8591 cells (Additional file 7: Figure S3). These results suggest that LGR5 ${ }^{+}$ cells possess stronger tumorigenicity in vivo than LGR5 cells, and LGR5 may thus be implicated in the malignancy, invasiveness and stemness of glioma.

\section{Relationship between LGR5 and reported CSC markers and stem cell genes}

The expression levels of CD133, CD44, CD24, CD90 and EpCAM were examined in LGR5 ${ }^{+}$and $\mathrm{LGR}^{-} \mathrm{U} 251$ and 8591 glioma cells by FCM. We found that $\mathrm{LGR} 5^{+}$glioma cells possessed considerably enhanced expression of CD133, CD44, CD90, CD24, and EpCAM (Fig. 3a). 
a

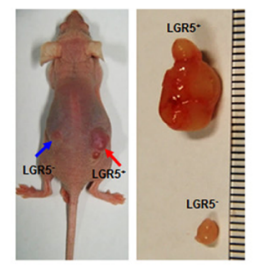

C

C
LGR5



b

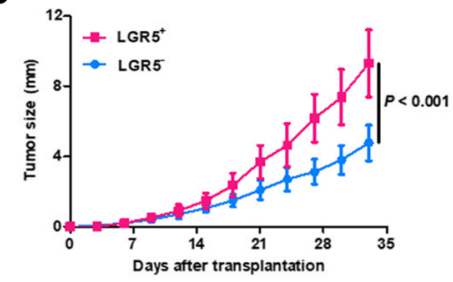

d $=\mathrm{LGRS}^{+}$

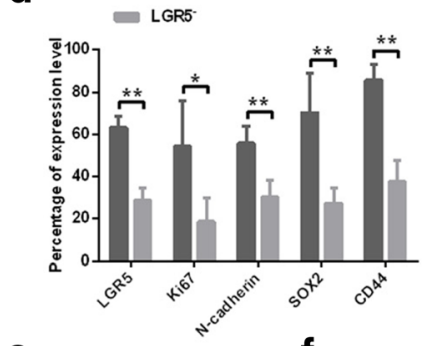

e
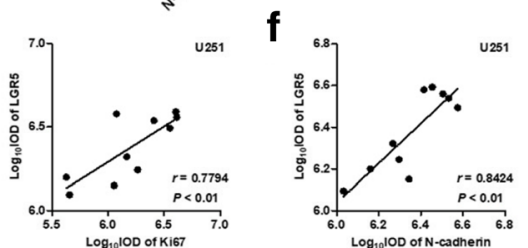

g

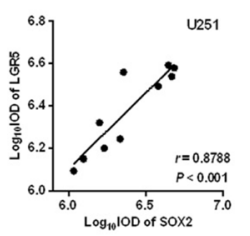

h
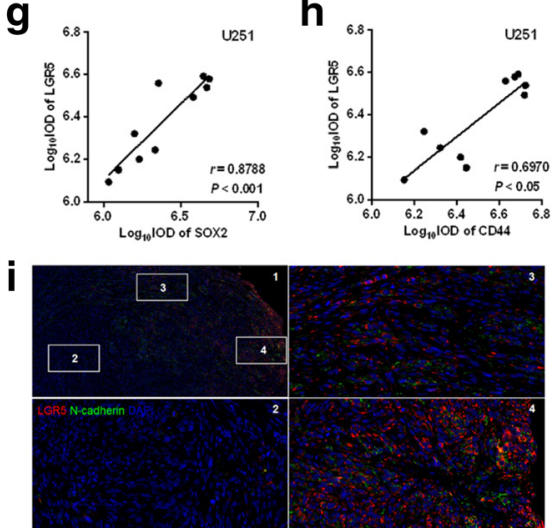

Fig. 2 Stemness properties of LGR5 ${ }^{+}$U251 cells in vivo. a Xenografts produced by LGR5 ${ }^{+}$and LGR5 $5^{-} 251$ cells. b The subcutaneous xenografts growth curve generated by LGR5 $5^{+}$and $L G R 5^{-}$U251 cells in BALB/c-nu mice (two-way ANOVA, $P<0.001$ ). Data are shown as the mean \pm SD $\left(\mathrm{LGR5}^{+} \mathrm{U} 251: n=5, \mathrm{LGR5}^{-} \mathrm{U} 251: n=5\right)$. c IHC staining of LGR5, Ki67, N-cadherin, CD44 and SOX2 expression in LGR5 ${ }^{+}$xenografts and LGR5 xenografts (magnification $\times 200$ ). $\mathbf{d}$ The percentage of positive expression of LGR5, Ki67, N-cadherin, CD44 and SOX2 in LGR5 ${ }^{+}$xenografts and LGR5 $^{-}$xenografts $(n=5$, Student $t$ test). Error bars represent the mean \pm SD. e The correlation between the levels of LGR5 and Ki67 by Spearman correlation analysis $(P<0.01, r=0.7794, n=10)$. $\mathbf{f}$ The correlation between the levels of LGR5 and $\mathrm{N}$-cadherin by Spearman correlation analysis $(P$ $<0.01, r=0.8424, n=10)$. $\mathbf{g}$ The correlation between the levels of LGR5 and SOX2 by Spearman correlation analysis $(P<0.001, r=0.8788, n=10)$. $\mathbf{h}$ The correlation between the levels of LGR5 and CD44 by Spearman correlation analysis $(P<0.05, r=0.6970, n=10)$. i Double-staining of LGR5 and N-cadherin in U251 subcutaneous xenografts. The xenografts edge ( 3 and 4) showed many positive cells expressed both LGR5 and Ncadherin, while there was few positive cells in inside xenografts (section "Methods"). Scale bar $=100 \mu \mathrm{m} .{ }^{*}, P<0.05 ;{ }^{* *}, P<0.01 ;{ }^{* *}, P<0.001$

Conversely, LGR5 ${ }^{-}$glioma cells showed low expression of CD133, CD44, CD90, CD24, and EpCAM, while the expression of CD90 remained unchanged in $\mathrm{LGR}^{+}$and LGR5 $5^{-}$glioma cells (Fig. 3a).

To further reveal the relationship between LGR5 and the reported CSC markers in GSCs, we obtained enriched cells (U251-GSCs, 8591-GSCs) from U251 and 8591 glioma cells (Fig. 3b, top). Immunofluorescence double staining showed that LGR5 and SOX2 were co-expressed in both U251-GSCs and 8591-GSCs (Fig. 3b, bottom). Then, U251-GSCs and 8591-GSCs were infected with a lentiviral shRNA vector targeting LGR5 (shLGR5) and a control shRNA (shCtrl). The infection efficiency was determined by Western blot (Fig. 3c) and FCM (Additional file 8: Figure S4). We determined that the expression levels of CSC markers CD133, CD44, 




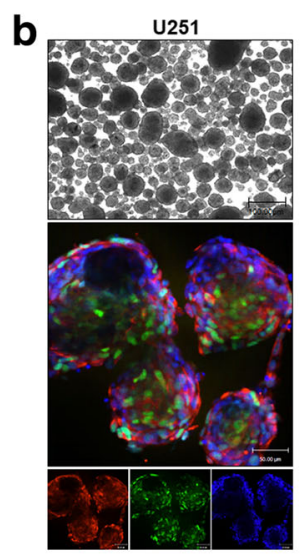

LGR5-SOX2-DAPI

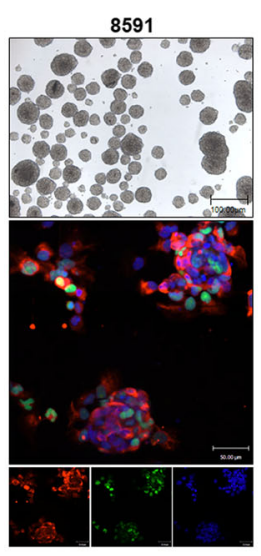

LGR5-SOX2-DAPI



Fig. 3 Relationship between LGR5 and reported CSCs markers and stem cell genes. a Analyses of CD133, CD44, CD90, CD24, and EpCAM expression in LGR5 $5^{+}$and $\mathrm{LGR5}^{-} \mathrm{U} 251$ glioma cells and in $\mathrm{LGR5}^{+}$and LGR5 8591 human primary glioma cells by FCM. b U251-GSCS and $8591-$ GSCs from U251 and 8591 human primary glioma cells through serum-free enrichment (top, scale bar $=100 \mu \mathrm{m}$ ) and immunofluorescence double staining for LGR5 and SOX2 (bottom, scale bar =50 $\mu \mathrm{m}$ ). c The expression levels of LGR5, CSCs markers (CD133, CD44, CD24 and EpCAM) and stem cell genes (SOX2, OCT4 and Nanog) in shLGR5 and shCtrl GSCs determined by Western blot (top). The protein intensity of these markers were determined by densitometric analysis and normalized to the relevant $\beta$-actin value. Error bars represent the mean \pm SD of three independent experiments (bottom). ${ }^{*}, P<0.05 ;{ }^{*}, P<0.01 ; * *, P<0.001$

CD24 and EpCAM in shLGR5 GSCs were significantly decreased compared with those of the shCtrl GSCs in both U251 and 8591 GSCs (Fig. 3c). Furthermore, stem cell genes Sox2, OCT4 and Nanog, critical factors favoring stem cell maintenance [10], were also examined. Although the expression of OCT4 remain unchanged, the expression levels of SOX2 and Nanog were obviously decreased in shLGR5 GSCs (Fig. 3c), suggesting that LGR5 might play a prominent role in the maintenance of GSCs.

\section{LGR5 promotes invasion, migration and EMT in GSCs}

Previous experiments have shown that $\mathrm{LGR}^{+}$cells possess stemness characteristics, particularly aggressive characteristics related to EMT. With the primary focus on strong invasion ability and $\mathrm{N}$-cadherin expression of $\mathrm{LGR}^{+}$cells, we explored the association between LGR5 and EMT in GSCs. The LGR5-overexpressed GSCs (Lenti-LGR5) and their control group (Lenti-GFP) were obtained by lentivirus transfection with U251-GSCs and 8591-GSCs. The transfection efficiency was determined by Western blot (Fig. 4c, d) and FACS (Additional file 8: Figure S4). Thus, invasion and migration assays were performed in shLGR5 and Lenti-LGR5 groups separately as well as in the controls (shCtrl and Lenti-GFP). We found that the invasion and migration capacities of shLGR5 GSCs were weakened as a result of LGR5 knockdown, whereas the invasion and migration capacities of Lenti-LGR5 GSCs were enhanced as a result of LGR5 overexpression (Fig. 4a, b).

To determine whether LGR5 is involved in EMT, we determined the expression levels of E-cadherin, $\mathrm{N}$-cadherin and Vimentin in the abovementioned transfected GSCs. The expression levels of the EMT markers were lower in the shLGR5 GSCs compared with those in the shCtrl GSCs and higher in the Lenti-LGR5 GSCs compared with those in the Lenti-GFP GSCs (Fig. 4c, d). These results indicate that LGR5 can promote the EMT of GSCs. 

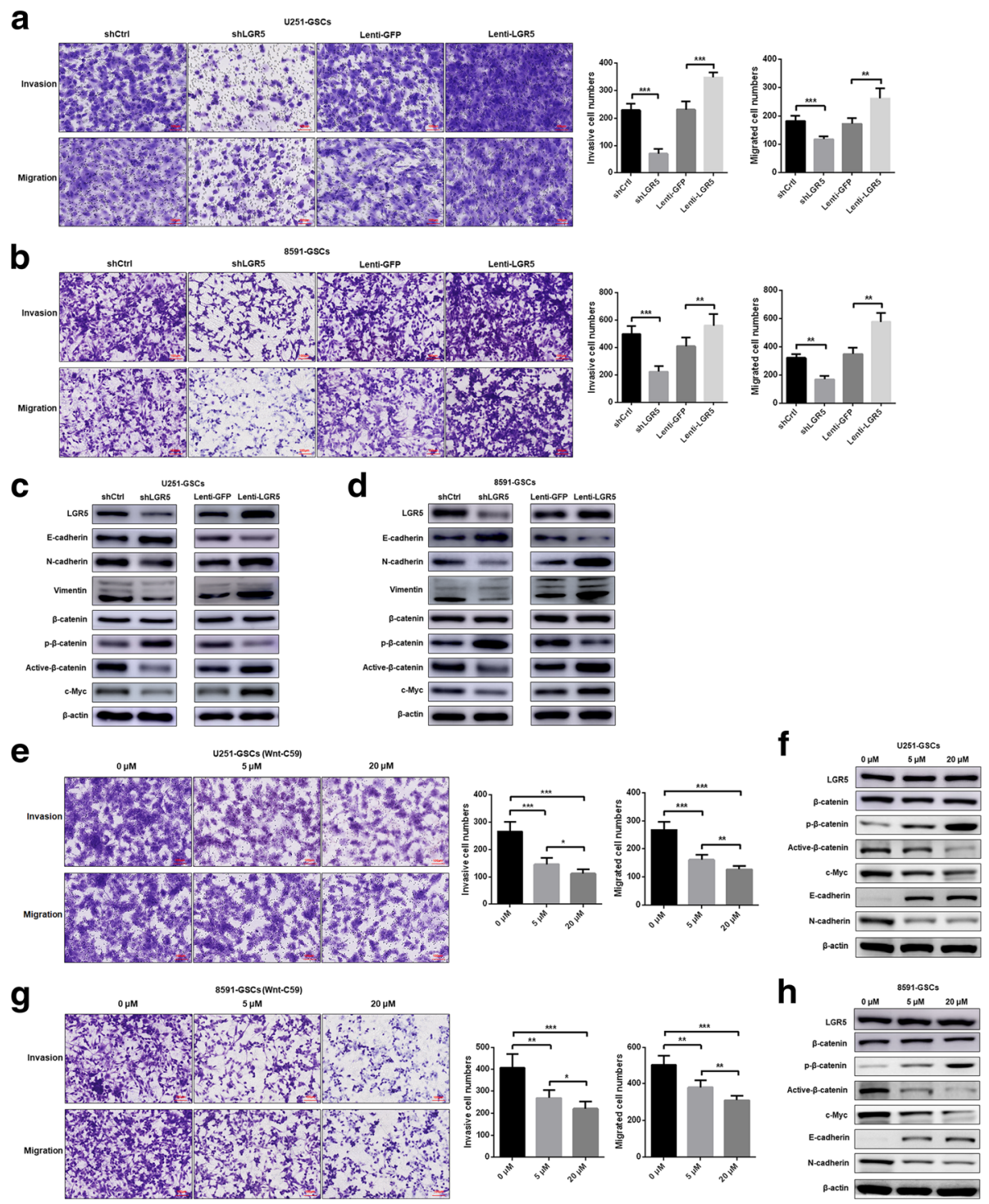

Fig. 4 Relationship between LGR5 and EMT and the Wnt/B-catenin pathway, and Wnt-C59 treatment in GSCs. a Images and statistical results in invasion assays $(P<0.001, n=3 ; P<0.001, n=3$, Student $t$ test for shLGR5 and Lenti-LGR5, respectively) and migration assays $(P<0.001, n=3 ; P<$ $0.01, n=3$, Student $t$ test for shLGR5 and Lenti-LGR5, respectively) for transfected U251 GSCs. $\mathbf{b}$ Images and statistical results in invasion assays ( $P$ $<0.001, n=3 ; P<0.01, n=3$, Student $t$ test for shLGR5 and Lenti-LGR5, respectively) and migration assays $(P<0.01, n=3 ; P<0.01, n=3$, Student $t$ test for shLGR5 and Lenti-LGR5, respectively) for transfected 8591 GSCs. c, d Western blot analyses of transfected U251 and 8591 GSCs for LGR5, EMT related genes and surrogate markers for the activated Wnt/ $\beta$-catenin pathway. e Images and statistical results of invasion assays $(P<0.001, n$ $=3 ; P<0.001, n=3 ; P<0.05, n=3$, one-way analysis of variance (ANOVA) for $0 \mu \mathrm{M}$ and $5 \mu \mathrm{M}, 0 \mu \mathrm{M}$ and $20 \mu \mathrm{M}$ and $5 \mu \mathrm{M}$ and $20 \mu \mathrm{M}$, respectively) and migration assays $(P<0.001, n=3 ; P<0.001, n=3 ; P<0.01, n=3$, one-way analysis of variance (ANOVA) for $0 \mu \mathrm{M}$ and $5 \mu \mathrm{M}, 0 \mu \mathrm{M}$ and $20 \mu \mathrm{M}$ and $5 \mu \mathrm{M}$ and $20 \mu \mathrm{M}$, respectively) at $24 \mathrm{~h}$ after treated with Wnt-C59 in U251-GSCs. f Western blot analyses of U251 GSCs for LGR5, EMT related genes and surrogate markers for the activated Wnt/ $\beta$-catenin pathway at $48 \mathrm{~h}$ after treated with Wnt-C59. $\mathbf{g}$ Images and statistical results of invasion assays $(P<0.01, n=3 ; P<0.001, n=3 ; P<0.05, n=3$, one-way analysis of variance (ANOVA) for $0 \mu \mathrm{M}$ and $5 \mu \mathrm{M}, 0 \mu \mathrm{M}$ and $20 \mu \mathrm{M}$ and $5 \mu \mathrm{M}$ and $20 \mu \mathrm{M}$, respectively) and migration assays $(P<0.01, n=3 ; P<0.001, n=3 ; P<0.01, n=3$, one-way analysis of variance (ANOVA) for $0 \mu \mathrm{M}$ and $5 \mu \mathrm{M}, 0 \mu \mathrm{M}$ and $20 \mu \mathrm{M}$ and $5 \mu \mathrm{M}$ and $20 \mu \mathrm{M}$, respectively) at $24 \mathrm{~h}$ after treated with Wnt-C59 in 8591-GSCs. h Western blot analyses of 8591 GSCs for LGR5, EMT related genes and surrogate markers for the activated Wnt/B-catenin pathway at $48 \mathrm{~h}$ after treated with Wnt-C59. Scale bar = $100 \mu \mathrm{m}$. All bars are represented as mean \pm SD from triplicate wells. ${ }^{* *}, P<0.001 ;{ }^{* *}, P<0.01 ;{ }^{*}, P<0.05$

\section{LGR5 promotes EMT by activating Wnt/ $\beta$-catenin signaling in GSCs}

The active form of $\beta$-catenin (active $\beta$-catenin) which is free from degraded by phosphorylation and proteolytic enzymes represents the activated Wnt/ $\mathrm{W}$-catenin pathway $[36,37]$. We verified the relationship between LGR5 and $\beta$-catenin phosphorylation. As shown in Fig. $4 \mathrm{c}$ and $d$, the expression level of phospho- $\beta$-catenin Ser33/37/Thr41 
(p- $\beta$-catenin) was up-regulated and that of Non-phospho (Active) $\beta$-catenin Ser33/37/Thr41 (Active- $\beta$-catenin) was down-regulated in the shLGR5 GSCs, while the expression level of total $\beta$-catenin remained unaltered. Conversely, Lenti-LGR5 GSCs showed decreased expression of $\mathrm{p}-\beta$-catenin, increased expression of Active- $\beta$-catenin and unchanged expression of total $\beta$-catenin compared with the Lenti-GFP GSCs (Fig. 4c, d). As expected, the expression of c-Myc, a downstream target gene of the Wnt/ $\beta$-catenin pathway [38], was proved to be modulated by LGR5 (Fig. 4c, d). These results confirmed that LGR5 can activate the $\mathrm{Wnt} / \beta$-catenin pathway by inhibiting $\beta$-catenin phosphorylation in GSCs.

Based on the previous results indicating that LGR5 can promote EMT, we assumed that LGR5 promotes EMT by activating the $\mathrm{Wnt} / \beta$-catenin pathway. To test this assumption, we treated U251 and 8591 GSCs with the Wnt/ $\beta$-catenin inhibitor Wnt-C59 and found that the expression levels of both the Wnt/ $\beta$-catenin pathway and EMT markers were inhibited in U251 and 8591 GSCs except for LGR5 expression level (Fig. 4f, h). Likewise, the invasion and migration capacities of U251 and 8591 GSCs were both decreased in a dose-dependent manner (Fig. 4e, g). Overall, we demonstrated that LGR5 can promote EMT and enhance the invasion and migration capacity of GSCs by activating the Wnt/ $\beta$-catenin pathway (Fig. 6h).

\section{Knockdown of LGR5 and Wnt-C59 treatment inhibit growth of intracranial orthotopic xenografts and prolong overall survival (OS) of xenograft mice}

Gliomas are different from other tumors due to the presence of the blood-brain barrier. Therefore, it is essential to treat glioma in the intracranial orthotopic xenograft model. We thus conducted a therapeutic experiment using the intracranial xenograft model in three groups, shCtrl, shLGR5 and shCtrl by Wnt-C59 treatment (Wnt-C59), to explore the role of LGR5 in vivo. The intracranial tumors induced by U251-GSCs were assessed on days 12, 25 and 39 (Fig. 5a). The corresponding tumor growth curve showed that tumors in the shLGR5 $(n=5)$ and Wnt-C59 $(n=5)$ groups were significantly inhibited, in contrast to those in the shCtrl group $(n=5$; Fig. $5 \mathrm{~b}, P<0.001, P<$ 0.001 , respectively). The median survival times (MS) of mice in the shLGR5 and Wnt-C59 groups were $62 \pm$ 4.45 days and $57 \pm 1.83$ days, respectively, while in the shCtrl group, the MS was $49 \pm 2.15$ days. A log-rank test of the three groups revealed that the OS of the shLGR5 and Wnt-C59 mice was significantly prolonged compared with that of the shCtrl mice $(n=5$; Fig. $5 \mathrm{c}, P<0.01, P<0.01$, respectively). In addition, quantitative analysis of IHC was used to determine the expression of LGR5, Ki67, Active- $\beta$-catenin, SOX2 and CD44. The expression level of LGR5 was statistically significant between the shCtrl and shLGR5 groups $(P<0.001$, Fig. $5 \mathrm{~d})$ and between the shCtrl and Wnt-C59 groups $(P<0.001$, Fig. $5 d)$. Notably, the expression levels of Ki67, Active- $\beta$-catenin, SOX2 and CD44 in both the shLGR5 and Wnt-C59 groups were lower than those in the shCtrl group, whereas the expression levels of above markers showed no differences between the shLGR5 and Wnt-C59 groups (Fig. 5d). Furthermore, Spearman correlation tests verified that the expression of LGR5 was positively correlated with Active- $\beta$-catenin $(P<0.001, r=$ 0.8940, $n=10$, Fig. 5e) and Ki67 $(P<0.01, r=0.7803, n=$ 10, Fig. 5f), as well as SOX2 $(P<0.01, r=0.8182, n=10$, Fig. 5 g) and CD44 ( $P<0.01, r=0.8788, n=10$, Fig. 5 h), between the shLGR5 and shCtrl groups. Similar results were observed in 8591 GSCs (Fig. 5i-p). These results demonstrated that the inhibition of LGR5 could inhibit glioma growth and prolong the survival of the xenograft mice in vivo effectively, indicating that LGR5 might serve as a prospective therapeutic target for glioma.

\section{Expression levels of LGR5 correlate with WHO grades, Ki67 and N-cadherin}

The expression levels of LGR5, Ki67 and N-cadherin were determined in 268 human glioma specimens by IHC (Fig. 6a). Significant differences in LGR5 levels were observed between high-grade (WHO III and IV) and low-grade (WHO I and II) glioma as well as between WHO I and WHO II glioma $(P<0.001, P<0.05$, respectively, Fig. $6 \mathrm{~b})$. In addition, both Ki67 and N-cadherin levels increased with grade (Fig. 6b). Spearman correlation analyses were performed to determine whether LGR5 levels correlated with Ki67 and N-cadherin. Positive correlations were observed between the levels of LGR5 and Ki67 $(P<0.001, r=0.8528$, $n=268$, Fig. $6 \mathrm{c}$ ) and between the levels of LGR5 and N-cadherin $(P<0.001, r=0.7128, n=268$, Fig. $6 d)$. In addition, many glioma cells with both LGR5 and $\mathrm{N}$-cadherin expression were observed in the invasive edge of human glioma tissues, while the internal tumor rarely expressed LGR5 and N-cadherin (Fig. 6e). The HE image showed the tissue structure of tumor edge, consistent with the invasive edge in Fig. 6e, were more incompact compared with the inside tumor (Fig. 6f).

\section{Correlations of LGR5 levels with survival of glioma patients}

In view of the differences in LGR5 expression as well as the multifarious differences in high- and low-grade glioma [2], we divided all glioma patients into a low-grade glioma (LGG, WHO I and II) group and a high-grade glioma (HGG, WHO III and IV) group. The clinicopathologic characteristics of glioma patients and their correlations with LGR5 expression are summarized in Additional file 1: Table S1 and Additional file 2: Table S2. LGR5 expression levels were positively correlated with Ki67 and negatively correlated with isocitrate dehydrogenase 1 (IDH1) in both the HGG group and LGG 
a

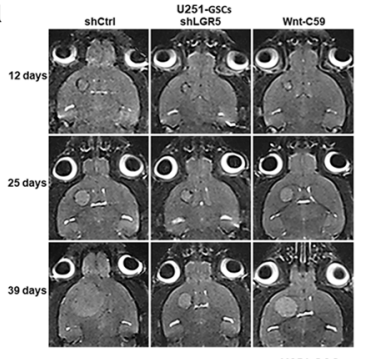

d
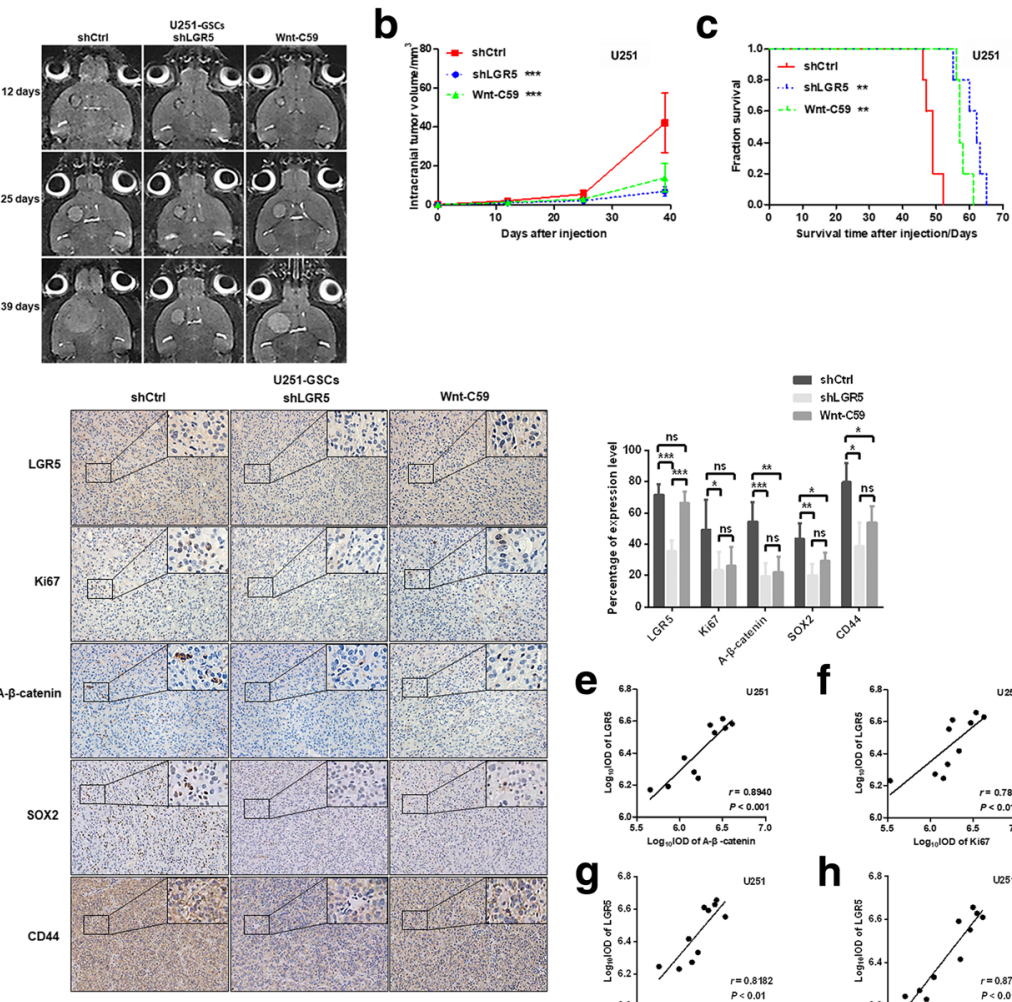



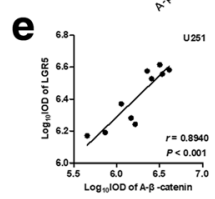

9
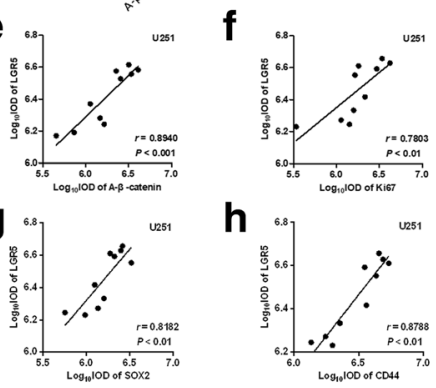

h

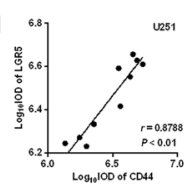

i
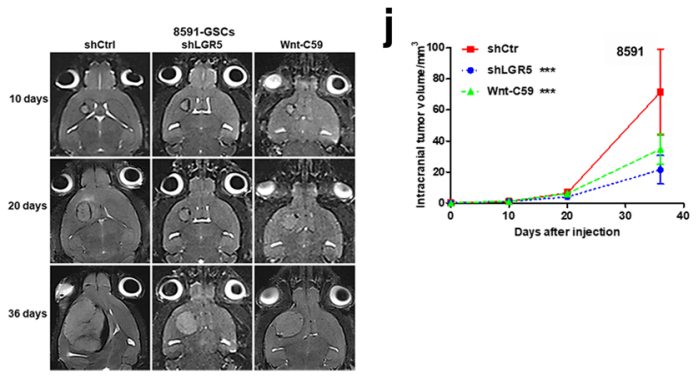

$\mathbf{k}$

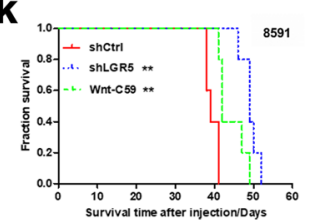

I

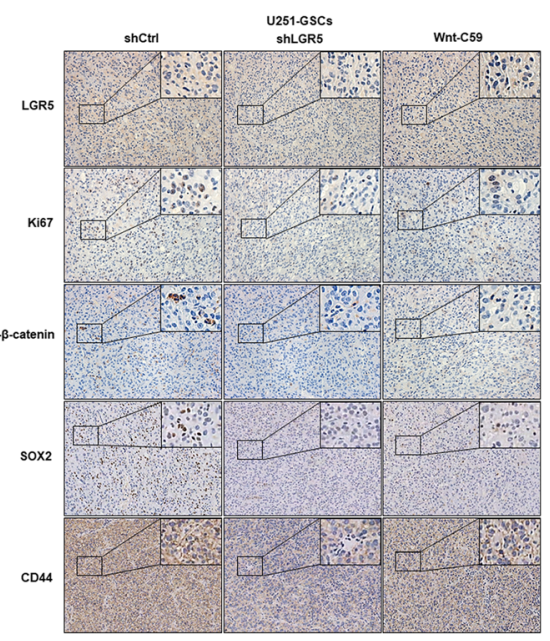

$\mathbf{m}$

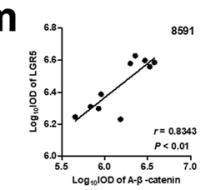

0



.

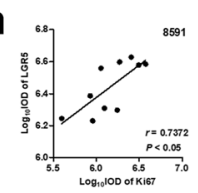

p

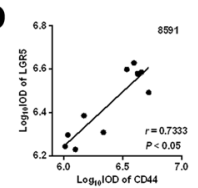

Fig. 5 (See legend on next page.) 




group (Additional file 1: Table S1 and Additional file 2: Table S2). Notably, although there was no statistical significance, patients with high expression of LGR5 (LGR5 ${ }^{\text {high }}$ ) in the HGG group tended to have more severe peritumoral edema $(P=0.079)$ (Additional file 1 : Table S1). Representative images of peritumoral edema in the HGG group and the LGG group are shown in Additional file 9: Figure S5.

Log-rank tests were performed to explore whether LGR5 expression level is significant with respect to the prognosis of glioma patients. In the HGG group, LGR $5^{\text {high }}$ patients had significantly lower progression-free survival (PFS) $(P=0.018)$ and OS $(P=0.028)$ compared with that of patients with low expression of LGR5 (LGR $5^{\text {low }}$ ). The median PFS and OS were 7.0 and 17.5 months for LGR $5^{\text {high }}$ patients and 10.5 and 23.0 months for LGR $5^{\text {low }}$ patients, respectively (Additional file 10: Table S3). In the LGG group, LGR $5^{\text {high }}$ patients had significantly lower RFS $(P=0.012)$ than LGR ${ }^{\text {low }}$ patients, while the OS was not statistically significant between LGR $5^{\text {high }}$ patients and LGR $5^{\text {low }}$ patients $(P=0.058)$. The median PFS of LGR $5^{\text {high }}$ patients was 23.5 months; the OS of LGR $5^{\text {high }}$ patients and the PFS and OS of LGR $5{ }^{\text {low }}$ patients in the LGG group were not available (Additional file 11: Table S4).

Furthermore, in the HGG group, univariate analysis revealed that LGR5 expression, peritumoral edema and chemoradiotherapy were correlated with worse PFS (Additional file 10: Table S3). Multivariate Cox regression analysis demonstrated that LGR5 expression and chemoradiotherapy were independent indicators of postoperative recurrence (Table 1). Moreover, LGR5 expression, the Karnofsky Performance Scale (KPS), IDH1 mutation and chemoradiotherapy were associated with worse OS according to the log-rank test (Additional file 10: Table S3). Multivariate analysis showed that LGR5 expression, IDH1 mutation and chemoradiotherapy were independent indicators of OS (Table 1). In the LGG group, IDH1 mutation and chemoradiotherapy significantly decreased both RFS and OS, whereas LGR5 expression was only related with shorter PFS but not OS (Additional file 11: Table S4). In multivariate analysis, LGR5 expression and chemoradiotherapy were key factors resulting in shorter PFS (Table 1).

\section{Discussion}

Most glioma cases tend to show recurrence and poor prognosis, which are believed to be closely related to the existence of GSCs [10-12]. It is necessary to identify GSCs and find GSC-related therapeutic targets to improve glioma outcomes. Our findings showed that LGR5 can serve as a functional marker of GSCs with strong stemness properties. The finding that LGR5 proportion was much higher in enriched cells than that in parent cells suggests that LGR5 conformed to the typical features of a GSC marker. Cell proliferation assays and clone-formation assays revealed stronger malignant potency of $\mathrm{LGR}^{+}{ }^{+}$cells. $\mathrm{LGR}^{+}$cells were more resistant to TMZ than LGR5 ${ }^{-}$cells. Invasion and migration assays showed that LGR5 ${ }^{+}$cells were more invasive and migratory. Moreover, the tumors produced by $\mathrm{LGR}^{+}$cells were larger, more invasive and malignant in subcutaneous transplantation experiments than those produced by LGR $5^{-}$cells. These results strongly indicate that LGR $5^{+}$ glioma cells possess a stronger stem-like phenotype.

Several CSC markers have been reported in glioma, such as CD133, CD24, CD90, CD44 and EpCAM [10, 21, 22]. Our FCM analysis revealed that $\mathrm{LGR}^{+}$glioma cells displayed significantly higher expression of these CSCs markers, indicating that $\mathrm{LGR}^{+}$glioma cells have stronger stem cell characteristics than LGR $5^{-}$cells. Furthermore, the conjecture that the expression of these CSCs markers in GSCs were modulated by LGR5 was confirmed by results indicating reduced expression of CD133, CD24, CD44 and EpCAM in LGR5 knockdown GSCs. In addition, although OCT4 expression did not change, we found that the acknowledged stem cell genes SOX2 and Nanog were obviously regulated by LGR5. These results suggest that LGR5 may serve as a vital and advanced GSC marker. 


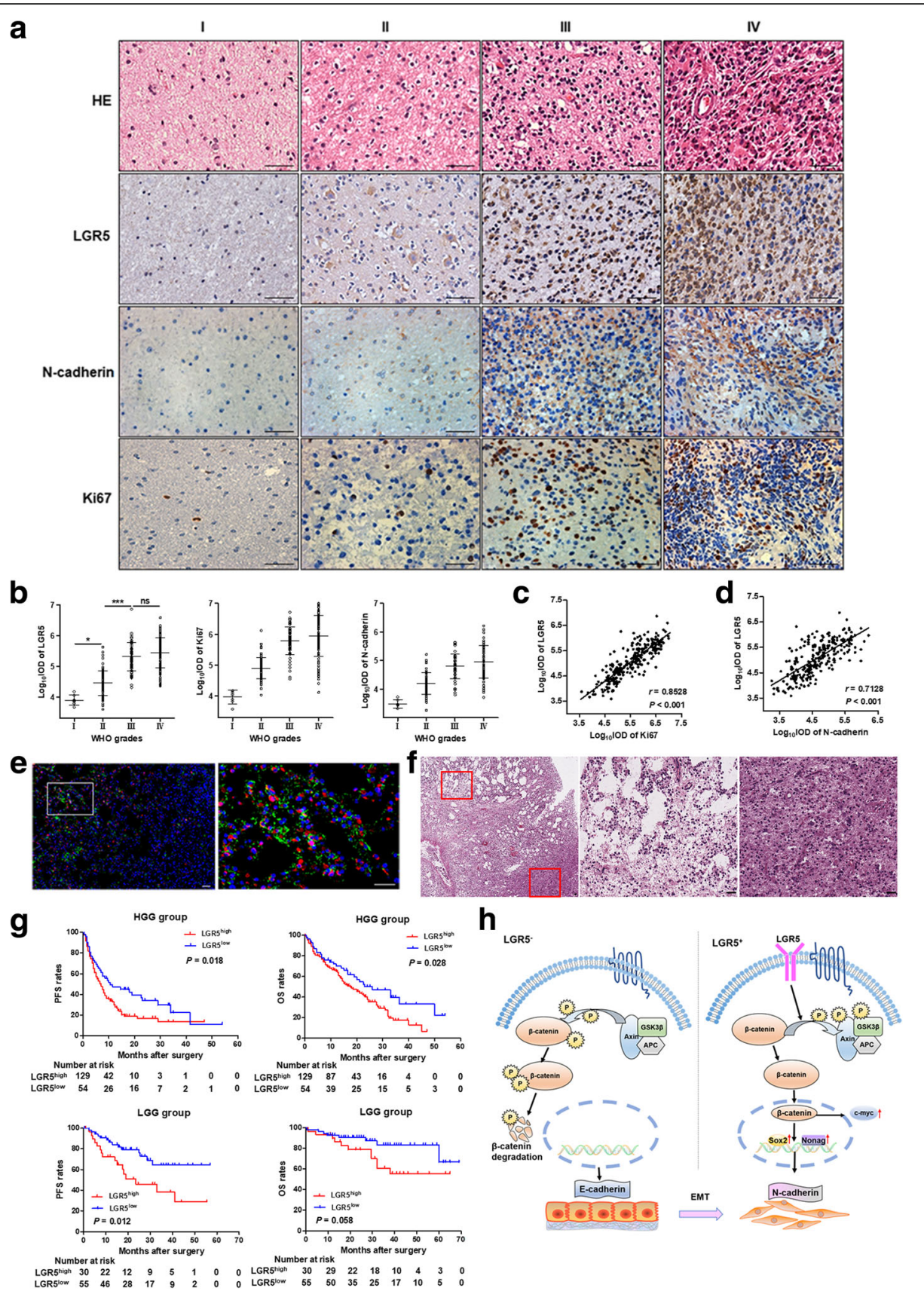

Fig. 6 Immunohistochemistry analyses of human glioma specimens. a HE and LGR5, Ki67 and N-cadherin staining of human glioma specimens from four patients in four WHO grades. Scale bar $=100 \mu \mathrm{m}$. b Quantitative analyses of LGR5, Ki67 and N-cadherin in four WHO grades (WHO I and II, $n=85$; WHO III and IV, $n=183$, Student $t$ test). Data are showed as mean \pm SD. c Spearman correlation analyses of LGR5 and Ki67 $(P<0.001, r=0.8528, n=$ 268). d Spearman correlation analyses of LGR5 and N-cadherin $(P<0.001, r=0.7128, n=268)$. e LGR5 and N-cadherin staining in glioma tissues. Many glioma cells with high expression of LGR5 and N-cadherin were mostly observed in the invasive edge of tumor (left of 1st picture) compared with the interior of tumor (right of 1st picture). Scale bar $=100 \mu \mathrm{m}$. f The HE staining of the glioma tissues. The structure of tumor edge (left top), consistent with the invasive edge of Fig. 6e, were more incompact compared with the inside tumor (right bottom). $\mathbf{g}$ PFS and OS months in low-grade glioma (LGG) group and high-grade glioma (HGG) group according to LGR5 expression in the glioma specimens. ***, $P<0.001$; ${ }^{*}, P<0.05$; ns, not significant. h A schema diagram displaying the role of LGR5 in regulating Wnt, and EMT in glioma stem cells. Based on the findings of this study, LGR5 could activate Wnt pathway by promoting $\beta$-catenin dephosphorylation and result in EMT eventually 
Table 1 Multivariate analysis of factors predicting prognosis

\begin{tabular}{lcc}
\hline Variable & Hazard Ratio $(95 \% \mathrm{Cl})$ & $P$ value \\
\hline Progression-free survival in the HGG group & \\
Chemoradiotherapy & $0.58(0.41-0.85)$ & $\mathbf{0 . 0 0 5}$ \\
LGR5 & $1.64(1.10-2.43)$ & $\mathbf{0 . 0 1 2}$ \\
Peritumoral edema & $1.28(0.88-1.85)$ & 0.193 \\
Overall survival in the HGG group & \\
Chemoradiotherapy & $0.53(0.32-0.87)$ & $\mathbf{0 . 0 1 9}$ \\
LGR5 & $1.65(1.07-2.53)$ & $\mathbf{0 . 0 2 4}$ \\
IDH1 mutation & $0.56(0.34-0.93)$ & $\mathbf{0 . 0 2 4}$ \\
KPS & $0.71(0.49-1.04)$ & 0.080 \\
Progression-free survival in the LGG group & \\
Chemoradiotherapy & $0.18(0.05-0.62)$ & $\mathbf{0 . 0 0 7}$ \\
LGR5 & $2.56(1.20-5.45)$ & $\mathbf{0 . 0 1 5}$ \\
IDH1 mutation & $0.48(0.23-1.02)$ & 0.056 \\
Overall survival in the LGG group & \\
IDH1 mutation & $0.26(0.09-0.74)$ & $\mathbf{0 . 0 1 2}$ \\
LGR5 & $2.08(0.80-5.42)$ & 0.136 \\
Chemoradiotherapy & $0.00(0.00-2.03)$ & 0.969 \\
\hline
\end{tabular}

Abbreviation: $H G G$ high-grade glioma, LGG low-grade glioma, $L G R 5$ leucine-rich repeat-containing G protein-coupled receptor 5, KPS karnofsky performance scale, $I D H 1$ isocitrate dehydrogenase $1, C l$ confidence interval, $N A$ not available. Bold and italic value represents $P<0.05$ that are considered statistically significant

LGR5 has been reported as a proliferative factor in multiple tumors, with the ability to regulate cell cycle proteins, such as p27, pRb and CyclinD1 [36, 39]. In addition, studies have shown that the expression of LGR5 is important for the cloning and tumorigenic abilities of glioma cells [30,33]. Similarly, our research showed that $\mathrm{LGR}^{+}$cells possessed significantly stronger stem cell characteristics in cell proliferation, cloning, tumorigenicity, and even drug resistance. However, the role of LGR5 in glioma invasion remains unclear, particularly for GSCs. Recently, increasing evidences indicated that the contribution of GSCs to tumor recurrence is most likely attributed to its EMT phenotype $[8,19,40]$. Therefore, it is important to find the primary effector molecules and signaling pathways that driven EMT in GSCs.

In this study, for the first time, we found that LGR5 was associated with the invasiveness of GSCs in vitro and in vivo. LGR5 could significantly affect invasion and migration in GSCs. The tumors derived from LGR5 ${ }^{+}$cells possessed higher invasive phenotype. Moreover, the epithelial marker E-cadherin and the mesenchymal markers including $\mathrm{N}$-cadherin and vimentin were regulated by LGR5 in GSCs, suggesting that LGR5 is a key effector molecule that drives the EMT of GSCs. Then, we explored the mechanism of glioma invasion and migration regulated by LGR5. Although LGR5 has been reported to be involved in the Wnt/ $\beta$-catenin pathway [25], it has not been verified in glioma. We demonstrated that LGR5 can activate the
Wnt/ $\beta$-catenin pathway by inhibiting the phosphorylation of $\beta$-catenin in vitro and in vivo. Furthermore, Wnt-C59, a Wnt/ $\beta$-catenin pathway inhibitor, inhibited both EMT and the Wnt/ $\beta$-catenin pathway as well as the invasion and $\mathrm{mi}-$ gration of glioma cells. Based on the abovementioned loop treatment verification, we can conclude that LGR5 can promote glioma invasion, migration and EMT by activating the Wnt/B-catenin pathway (Fig. 6h).

Notably, we found that both LGR5 knockdown and Wnt-C59 significantly inhibited the growth of tumors and prolonged the survival time of mice in intracranial orthotopic xenograft models. Additionally, the treatment effect of orthotopic xenografts tended to be more distinct in the LGR5 knockdown group, suggesting the possibility of the involvement of LGR5 with other tumorigenesis-related pathways. IHC analysis of orthotopic xenografts revealed that the expression levels of Ki67 and Active- $\beta$-catenin were significantly decreased in both the LGR5 knockdown and Wnt-C59 groups. Furthermore, LGR5 expression was significantly correlated with Ki67 and Active- $\beta$-catenin. These results illustrate that LGR5 may be a potential target for blocking EMT in GSCs.

Similar to the results of the animal experiment in vivo, LGR5 expression level was proved to be positively correlated with WHO grade, Ki67 and N-cadherin by quantitative analysis of IHC of human glioma tissues. In addition, $\mathrm{LGR}^{+} \mathrm{N}$-cadherin ${ }^{+}$cells were mostly observed in the invasive front of human glioma tissues, while glioma cells inside tumors rarely expressed LGR5 and N-cadherin, suggesting a correlation between the two markers and their important role in the high invasion of glioma.

Furthermore, IDH1 mutation, a favorable predictor of prognosis [41], was confirmed to be negatively correlated with LGR 5 by chi-square tests in both HGG and LGG group. This result indicated that IDH1 mutation in LGR $5^{\text {high }}$ glioma occurred less frequently than that in LGR $5^{\text {low }}$ glioma, suggesting that glioma patients with high expression of LGR5 may have a poor prognosis. However, the relationship between LGR5 and IDH1 must be further explored. Although LGR5 has been identified as a prognostic factor in many tumors [30,33, 42, 43], there are few studies on the prognostic value of LGR5 in glioma. The prognostic value of LGR5 in glioma need more evidence to confirm. To verify the value of LGR5 in estimating glioma prognosis, univariate and multivariate analysis were performed. The results revealed that LGR5 expression was an independent indicator of postoperative recurrence in both the HGG group and LGG group; moreover, LGR5 expression was also an independent indicator of OS in the HGG group, consistent with the results indicating that LGR5 knockdown prolonged the survival of nude mice in vivo. However, in the LGG group, LGR5 expression tended to be related to poor OS in univariate analysis $(P=0.058)$. This result may be attributed to the fact that 
the review period of this study was not sufficiently long. Additionally, LGR5 expression tended to relate to peritumoral edema in HGG group, although the difference was not statistically significant $(P=0.079)$. Overall, our finding that LGR ${ }^{\text {high }}$ expression identifies a subset of glioma patients with a poorer survival profile than LGR $5^{\text {low }}$ expression might provide aid for clinicians in determining the prognosis of glioma patients.

\section{Conclusions}

Our study demonstrates that LGR5 can serve as a functional GSC marker, drive GSCs EMT and by activating the $\mathrm{Wnt} / \beta$-catenin pathway in vitro and in vivo, and predicts glioma recurrence and poor prognosis. Thus, we believe that further studies targeting LGR5 will provide novel therapeutic approaches for treating GSCs.

\section{Additional files}

Additional file 1: Table S1. Association of LGR5 expression with clinicopathological features in HGG group. (XLS $10 \mathrm{~kb}$ )

Additional file 2: Table S2. Association of LGR5 expression with clinicopathological features in LGG group. (XLS $10 \mathrm{~kb}$ )

Additional file 3: Short tandem repeat (STR) profiling of U251. (PDF $2190 \mathrm{~kb}$ )

Additional file 4: Short tandem repeat (STR) profiling of 8591 . (PDF $2185 \mathrm{~kb}$ )

Additional file 5: Figure S1. Flow cytometry (FCM) analyses of LGR5 in glioma cells. (a) FCM analyses of LGR5 positive proportion in parent and enriched cells. (b) Fluorescence-activated cell sorting (FACS) of LGR5 ${ }^{+}$ cells in U251 glioma cells and 8591 primary glioma cells. (PPTX 2348 kb)

Additional file 6: Figure S2. Stemness properties of $\mathrm{LGRS}^{+} 8591$ cells in vitro. (a) Cell proliferation assays of $\mathrm{LGRS}^{+}$and $\mathrm{LGRS}^{-} 8591$ cells $(P<$ $0.001, n=3$, two-way ANOVA). (b) Coloning sphere images and sphere numbers in the clone formation assay $(P<0.001, n=3$, Student $t$ test). Scale bar $=100 \mu \mathrm{m}$. (c) Drug resistance curve of TMZ in LGR5 ${ }^{+}$and LGR5 8591 cells. (d) Images and numbers of invasive cells in invasion assays (top, $P<0.001, n=3$, Student $t$ test) and images and numbers of migrated cells in migration assays (bottom, $P<0.001, n=3$, Student $t$ test). All data are represented as mean \pm SD from triplicate wells. ${ }^{* *}, P<0.001$, as compared to control. (PPTX $7790 \mathrm{~kb}$ )

Additional file 7: Figure S3. Stemness properties of $L G R 5^{+} 8591$ cells in vivo. (a) Xenografts produced by LGR5 ${ }^{+}$and LGR5 8591 cells. (b) The subcutaneous xenografts growth rate generated by $\mathrm{LGRS}^{+}$and $\mathrm{LGR5}^{-}$ 8591 cells in BALB/C-nu mice (two-way ANOVA, $P=0.001$ ). Data are shown as the mean $\pm \mathrm{SD}\left(\mathrm{LGR5}^{+}\right.$8591: $n=5, \mathrm{LGR5}^{-}$8591: $\left.n=5\right)$. (c) IHC staining of LGR5, Ki67, N-cadherin, SOX2and CD44 expression in LGR5 ${ }^{+}$ xenografts and LGR5 ${ }^{-}$xenografts (magnification $\times 200$ ). (d) The percentage of positive expression of LGR5, Ki67, N-cadherin, SOX2and CD44 in $\mathrm{LGR}^{+}$xenografts and LGR5 ${ }^{-}$xenografts $(n=5$, Student $t$ test). Error bars represent the mean $\pm S D$. (e) The correlation between the levels of LGR5 and Ki67 by Spearman correlation analysis $(P<0.05, r=0.7505, n=10)$. (f) The correlation between the levels of LGR5 and N-cadherin by Spearman correlation analysis $(P<0.001, r=0.8989, n=10)$. (g) The correlation between the levels of LGR5 and SOX2 by Spearman correlation analysis $(P<0.01, r=0.7939, n=10)$. (h) The correlation between the levels of LGR5 and CD44 by Spearman correlation analysis $(P<0.01, r=0.8182$, $n=10$ ). (i) Double-staining of LGR5 and N-cadherin in 8591 subcutaneous xenografts. The xenografts edge (section "3 and 4") showed many positive cells expressed both LGR5 and N-cadherin, while there was few positive cells in inside xenografts (section "Methods"). Scale bar $=100 \mu \mathrm{m}$. *, $P<0.05 ;{ }^{* *}, P<0.01 ;{ }^{* * *}, P<0.001$. (PPTX $6203 \mathrm{~kb}$ )
Additional file 8: Figure S4. The transfection efficiency of transfected GSCs. (a) The transfection efficiency of transfected U251 GSCS by FCM analyses. (b) The transfection efficiency of transfected U251 GSCs by FCM analyses. (PPTX $2671 \mathrm{~kb}$ )

Additional file 9: Figure S5. The representative T2W images of peritumoral edema in the HGG group and the LGG group. The degree of peritumoral edema was divided into two groups according to the following criteria: maximum diameter $>2 \mathrm{~cm}$, severe; maximum diameter $\leqq 2 \mathrm{~cm}$, slight/None. (PPTX $1049 \mathrm{~kb}$ )

Additional file 10: Table S3. Univariate analysis of factors associated with prognosis in the HGG group. (XLS $13 \mathrm{~kb}$ )

Additional file 11: Table S4. Univariate analysis of factors associated with prognosis in the LGG group. (XLS $12 \mathrm{~kb}$ )

\section{Abbreviations}

CSCs: Cancer stem cells; EMT: Epithelial-mesenchymal transition; EpCAM: Epithelial cell adhesion molecule; FACS: Fluorescence-activated cell sorting; FCM: Flow cytometry; GBM: Glioblastoma multiforme; GFAP: Glial fibrillary acidic protein; GSCs: Glioma stem cells; HGG: High-grade glioma; IDH1: Isocitrate dehydrogenase 1; IF: Immunofluorescence; IHC: Immunohistochemistry; KPS: Karnofsky performance scale; LGG: Low-grade glioma; LGR5: Leucine-rich repeat-containing G protein-coupled receptor 5; OS: Overall survival; PFS: Progression-free survival; STR: Short tandem repeat; TMZ: Temozolomide

\section{Acknowledgments}

We wish to thank Dr. Shanshan Wang and Dr. Yiqiang Zhou for helpful and valuable suggestions.

\section{Funding}

This work was supported by the CAMS Innovation Fund for Medical Sciences (CIFMS) (Nos. 2016-I2M-3-013, 2017-I2M-3-005).

\section{Availability of data and materials}

All data generated or analyzed during this study are included in this published article and its additional files.

\section{Authors' contributions}

$J H W, Y L R$ and JZ conceived and designed experiments. JZ and HQC performed experiments. JZ wrote the first draft of this article. JZ, HQC, PPZ, $\mathrm{MC}$ and FZ contributed to data analysis and discussed the results. All authors read and approved the final manuscript.

\section{Ethics approval and consent to participate}

The glioma tissue collection was approved by the Ethics Committee of Cancer Hospital, Chinese Academy of Medical Sciences (No. NCC2014G-12). Each patient has written an informed consent to use part of the tissue for scientific research. The Experimental Animal Ethics Committee of Cancer Hospital, Chinese Academy of Medical Sciences approved the use of laboratory animals (No. NCC2014A037)

\section{Consent for publication}

Not applicable.

\section{Competing interests}

The authors declare that they have no competing interests.

\section{Publisher's Note}

Springer Nature remains neutral with regard to jurisdictional claims in published maps and institutional affiliations.

Received: 26 February 2018 Accepted: 4 August 2018

Published online: 12 September 2018

\section{References}

1. Lara-Velazquez M, Al-Kharboosh R, Jeanneret S, Vazquez-Ramos C, Mahato D, Tavanaiepour D, Rahmathulla G, Quinones-Hinojosa A. Advances in brain tumor surgery for glioblastoma in adults. Brain Sciences. 2017;7:166.

2. Wen PY, Kesari S. Malignant gliomas in adults. N Engl J Med. 2008;359: 492-507. 
3. Eisemann T, Costa B, Strelau J, Mittelbronn M, Angel P, Peterziel H. An advanced glioma cell invasion assay based on organotypic brain slice cultures. BMC Cancer. 2018;18:103.

4. Iwadate Y. Epithelial-mesenchymal transition in glioblastoma progression. Oncol Lett. 2016;11:1615-20.

5. Paw I, Carpenter RC, Watabe K, Debinski W, Lo HW. Mechanisms regulating glioma invasion. Cancer Lett. 2015;362:1-7.

6. Hannah E, Mukherjee S. Targeting adaptive glioblastoma: an overview of proliferation and invasion. Neuro-Oncology. 2014;16:1575-84.

7. Mao P, Joshi K, Li J, Kim SH, Li P, Santana-Santos L, Luthra S, Chandran UR, Benos PV, Smith L. Mesenchymal glioma stem cells are maintained by activated glycolytic metabolism involving aldehyde dehydrogenase 1A3. Proc Natl Acad Sci U S A. 2013;110:8644-9.

8. Nakano I. Stem cell signature in glioblastoma: therapeutic development for a moving target. J Neurosurg. 2015;122:324-30.

9. Liebelt BD, Shingu T, Zhou X, Ren J, Shin SA, Hu J. Glioma Stem Cells: Signaling, Microenvironment, and Therapy. Stem Cells International,2016,(2016-1-6). 2016;2016:7849890

10. Elena C, Ana-Maria E, Ionela-Daniela P, Simona M, Cristiana T. Glioma Stem Cells and Their Microenvironments: Providers of Challenging Therapeutic Targets. Stem Cells International,2016,(2016-2-10). 2016;2016:1-20.

11. Neman J, Jandial R. Decreasing glioma recurrence through adjuvant cancer stem cell inhibition. Biologics Targets \& Therapy. 2010;4:157-62.

12. Huang Q, Zhang QB, Dong J, Wu YY, Shen YT, Zhao YD, Zhu YD, Diao Y, Wang AD, Lan Q. Glioma stem cells are more aggressive in recurrent tumors with malignant progression than in the primary tumor, and both can be maintained long-termin vitro. BMC Cancer. 2008:8:304.

13. Bessède $E$, Staedel C, Acuña Amador LA, Nguyen PH, Chambonnier L, Hatakeyama M, Belleannée G, Mégraud F, Varon C. Helicobacter pylor generates cells with cancer stem cell properties via epithelial-mesenchymal transition-like changes. Oncogene. 2013;33:4123-31.

14. Shuang ZY, Wu WC, Xu J, Lin G, Liu YC, Lao XM, Zheng L, Li S. Transforming growth factor- $\beta 1$-induced epithelial-mesenchymal transition generates ALDH-positive cells with stem cell properties in cholangiocarcinoma. Cancer Lett. 2014;354:320

15. Radisky DC, Labarge MA. Epithelial-mesenchymal transition and the stem cell phenotype. Cell Stem Cell. 2008;2:511-2.

16. Mani SA, Guo W, Liao MJ, Eaton EN, Ayyanan A, Zhou AY, Brooks M, Reinhard F, Zhang CC, Shipitsin M. The epithelial-mesenchymal transition generates cells with properties of stem cells. Cell. 2008;133:704-15.

17. Abell Amy N, Jordan Nicole V, Huang W, Prat A, Midland Alicia A, Johnson Nancy L, Granger Deborah A, Mieczkowski Piotr A, Perou Charles M, Gomez Shawn M. MAP3K4/CBP-regulated H2B acetylation controls epithelialmesenchymal transition in trophoblast stem cells. Cell Stem Cell. 2011:8:525

18. Rhim AD, Mirek ET, Aiello NM, Maitra A, Bailey JM, Mccallister F, Reichert M, Beatty GL, Rustgi AK, Vonderheide RH. EMT and dissemination precede pancreatic tumor formation. Cell. 2012;148:349-61.

19. Abell AN, Johnson GL. Implications of mesenchymal cells in Cancer stem cell populations: relevance to EMT. Curr Pathobiol Rep. 2014;2:21-6.

20. Brescia P, Richichi C, Pelicci G. Current strategies for identification of glioma stem cells: adequate or unsatisfactory? Journal of Oncology,2012,(2012-523). 2012;2012:376894.

21. Song Y, Jang J, Shin TH, Bae SM, Kim JS, Kim KM, Myung SJ, Choi EK, Seo HR. Sulfasalazine attenuates evading anticancer response of CD133-positive hepatocellular carcinoma cells. J Exp Clin Cancer Res. 2017:36:38.

22. Xiao W, Gao Z, Duan Y, Yuan W, Ke Y. Notch signaling plays a crucial role in cancer stem-like cells maintaining stemness and mediating chemotaxis in renal cell carcinoma. J Exp Clin Cancer Res. 2017;36:41.

23. Lottaz C, Beier D, Meyer K, Kumar P, Hermann A, Schwarz J, Junker M Oefner PJ, Bogdahn U, Wischhusen J. Transcriptional profiles of CD133+ and CD133- glioblastoma-derived cancer stem cell lines suggest different cells of origin. Cancer Res. 2010;70:2030-40.

24. Beier D, Hau P, Proescholdt M, Lohmeier A, Wischhusen J, Oefner PJ, Aigner L, Brawanski A, Bogdahn U, Beier CP. CD133(+) and CD133(-) glioblastomaderived cancer stem cells show differential growth characteristics and molecular profiles. Cancer Res. 2007:67:4010.

25. Barker N, Huch M, Kujala P, Wetering MVD, Snippert HJ, Es JHV, Sato T, Stange DE, Begthel H, Born MVD. Lgr5 +ve stem cells drive self-renewal in the stomach and build long-lived gastric units in vitro. Cell Stem Cell. 2010;6:25.
26. Choi YJ, Kim N, Lee HS, Park SM, Ji HP, Yoon H, Shin CM, Park YS, Kim JW, Dong HL. Expression of leucine-rich repeat-containing G-protein coupled receptor 5 and CD44: potential implications for gastric Cancer stem cell marker. Journal of Cancer Prevention. 2016;21:279-87.

27. Wang B, Chen Q, Cao Y, Ma X, Yin C, Jia Y, Zang A, Fan W. LGR5 is a gastric Cancer stem cell marker associated with Stemness and the EMT signature genes NANOG, NANOGP8, PRRX1, TWIST1, and BMI1. PLoS One. 2016;11:e0168904.

28. Kobayashi S, Yamadaokabe H, Suzuki M, Natori O, Kato A, Matsubara K, Jau CY, Yamazaki M, Funahashi S, Yoshida K. LGR5-positive colon cancer stem cells interconvert with drug-resistant LGR5-negative cells and are capable of tumor reconstitution. Stem Cells. 2012;30:2631-44.

29. Takahashi H, Ishii H, Nishida N, Takemasa I, Mizushima T, Ikeda M, Yokobori T, Mimori K, Yamamoto H, Sekimoto M. Significance of Lgr5 +ve Cancer stem cells in the Colon and Rectum. Ann Surg Oncol. 2011;18:1166.

30. Parry PV, Engh JA. Knockdown of LGR5 suppresses the proliferation of glioma cells in vitro and in vivo. Neurosurgery. 2014;74:N14.

31. Chen X, Wei B, Han X, Zheng Z, Huang J, Liu J, Huang Y, Wei H. LGR5 is required for the maintenance of spheroid-derived colon cancer stem cells. Int J Mol Med. 2014;34:35.

32. Xi HQ, Cai AZ, Wu XS, Cui JX, Shen WS, Bian SB, Wang N, Li JY, Lu CR, Song $Z$. Leucine-rich repeat-containing G-protein-coupled receptor 5 is associated with invasion, metastasis, and could be a potential therapeutic target in human gastric cancer. Br J Cancer. 2014;110:2011.

33. Nakata S, Campos B, Bageritz J, Bermejo JL, Becker N, Engel F, Acker T, Momma S, Herold-Mende C, Lichter P. LGR5 is a marker of poor prognosis in glioblastoma and is required for survival of brain cancer stem-like cells. Brain Pathol. 2013;23:60-72.

34. Zhou Y, Jin G, Mi R, Dong C, Zhang J, Liu F. The methylation status of the platelet-derived growth factor-B gene promoter and its regulation of cellular proliferation following folate treatment in human glioma cells. Brain Res. 2014:1556:57.

35. Macarthur KM, Kao GD, Chandrasekaran S, Alonsobasanta M, Chapman C, Lustig RA, Wileyto EP, Hahn SM, Dorsey JF. Detection of brain tumor cells in the peripheral blood by a telomerase promoter-based assay. Cancer Res. 2014;74:2152-9.

36. Vieira GC, Chockalingam S, Melegh Z, Greenhough A, Malik S, Szemes M, Ji HP, Kaidi A, Zhou L, Catchpoole D. LGR5 regulates pro-survival MEK/ERK and proliferative Wnt/ $\beta$-catenin signalling in neuroblastoma. Oncotarget. 2015;6: 40053-67.

37. Yan W, Peng P, Wang Z, Yu Z, Peng X, Geng D, Yang J, Yu R, Zhou X. $\beta$ catenin-mediated YAP signaling promotes human glioma growth. J Exp Clin Cancer Res. 2017:36:136.

38. Lim JH, Chun YS, Park JW. Hypoxia-inducible factor-1alpha obstructs a Wnt signaling pathway by inhibiting the hARD1-mediated activation of betacatenin. Cancer Res. 2008;68:5177-84.

39. Chen Q, Cao HZ, Zheng PS. LGR5 promotes the proliferation and tumor formation of cervical cancer cells through the Wnt/ 3 -catenin signaling pathway. Oncotarget. 2014;5:9092.

40. Zhang X, Zhang W, Mao XG, Zhen HN, Cao WD, Hu SJ. Targeting role of glioma stem cells for glioblastoma multiforme. Curr Med Chem. 2013;20(15):1974-84.

41. Patel SH, Poisson LM, Brat DJ, Zhou Y, Cooper L, Snuderl M, Thomas C, Franceschi AM, Griffith B, Flanders A. T2-FLAIR Mismatch, an Imaging Biomarker for IDH and 1p/19q Status in Lower Grade Gliomas: A TCGA/TCIA Project. Clinical Cancer Research. 2017;23(20):6078-85. clincanres.0560.2017

42. Hou MF, Chen PM, Chu PY. LGR5 overexpression confers poor relapse-free survival in breast cancer patients. BMC Cancer. 2018;18:219.

43. Gao F, Zhou B, Xu JC, Gao X, Li SX, Zhu GC, Zhang XG, Yang C. The role of LGR5 and ALDH1A1 in non-small cell lung cancer: Cancer progression and prognosis. Biochem Biophys Res Commun. 2015;462:91-8. 